ISSN 0103-5150

Fisioter. Mov., Curitiba, v. 26, n. 3, p. 647-655, jul./set. 2013

Licenciado sob uma Licença Creative Commons

\title{
Pé diabético: orientações e conhecimento sobre cuidados preventivos
}

\author{
Diabetic foot: orientations and knowledge about prevention care
}

\author{
Marcia Regina Cubas ${ }^{[a]}$, Odette Moura dos Santos ${ }^{[b]}$, Elis Marina Andrade Retzlaff ${ }^{[c]}$, \\ Helouíse Letícia Cristiano Telma ${ }^{[\mathrm{d}]}$, Iria Priscila Silva de Andrade ${ }^{[\mathrm{e}]}$, Auristela D. de Lima Moser ${ }^{[\mathrm{f}]}$, \\ Ana Rotília Erzinger ${ }^{[\mathrm{g}]}$
}

[a] Doutora em Enfermagem pelo Departamento de Enfermagem em Saúde Coletiva da Escola de Enfermagem da Universidade de São Paulo (USP), professora do Programa de Pós-Graduação em Tecnologia em Saúde da Pontifícia Universidade Católica do Paraná (PUCPR), Curitiba, PR - Brasil, e-mail: m.cubas@pucpr.br

[b] Enfermeira na Diagnósticos da América (DASA), Curitiba, PR - Brasil, e-mail: odettemoura@hotmail.com

[c] Enfermeira na Secretaria Municipal de Saúde de Rebouças, Rebouças, PR - Brasil, e-mail: elis.marina@hotmail.com

[d] Enfermeira do Colégio Bom Jesus, São José dos Pinhais, PR - Brasil, e-mail: helouise_le@hotmail.com

[e] Enfermeira da Home-care Confiance, Curitiba, PR - Brasil, e-mail: prizoka_andrade@hotmail.com

[f] Doutora em Engenharia de Produção pela Universidade Federal de Santa Catarina (UFSC), professora do Programa de Pós-Graduação em Tecnologia em Saúde da Pontifícia Universidade Católica do Paraná (PUCPR), Curitiba, PR - Brasil, e-mail: auristela.lima@pucpr.br

[g] Mestre em Educação pela Pontifícia Universidade Católica do Paraná (PUCPR), professora do Curso de Enfermagem da Pontifícia Universidade Católica do Paraná (PUCPR), Curitiba, PR - Brasil, e-mail: ana.erzinger@pucpr.br

\section{Resumo}

Introdução: 0 pé diabético é considerado uma complicação do Diabete mellitus e a maior causa de amputações de membros inferiores. Para evitar seu aparecimento são necessárias orientações de medidas preventivas e autocuidado do portador. Objetivos: Verificar o conhecimento dos usuários do programa de diabetes acerca de cuidados preventivos ao pé diabético, identificar as orientações que o paciente recebe quanto à prevenção, e observar a aderência aos procedimentos de autocuidado preventivos. Materiais e métodos: Pesquisa exploratória de campo, envolvendo 40 diabéticos do tipo 2 e cinco enfermeiros. Foi realizado exame físico dos pés dos diabéticos e uma entrevista estruturada com os enfermeiros. Os dados foram organizados e analisados por estatística descritiva. Resultados e discussão: No exame físico verificaram-se grau de mobilidade comprometido em 52,5\% dos participantes; uso inadequado de calçados em $85 \%$; retirada 
de cutículas em 62,5\%. Não houve diferenças significativas na perfusão entre os pés direito e esquerdo, entretanto houve presença de micoses e rachaduras. Pontos de alta pressão apresentaram menores sensibilidades. As orientações fornecidas pelos enfermeiros são variáveis, todos afirmam orientar sobre o uso de calçados e corte de unhas; entretanto, não se verifica adesão a esses itens e faltam orientações importantes como o exame diário dos pés. Conclusão: Os itens com menor adesão são os mais simples e passíveis de correção. Fazem-se necessários adequada avaliação e acompanhamento individual levando em consideração o grau de conhecimento e a facilidade para processar as informações. Uma ação multiprofissional poderia potencializar as orientações e aumentar a aderência às mesmas.

Palavras-chave: Diabetes mellitus. Pé diabético. Prevenção e controle. Prevenção.

\section{Abstract}

Introduction: Diabetic foot is considered a complication of Diabetes mellitus and the major cause of lower limb amputations. To avoid its appearance orientations are needed for preventive measures and patient self-care. objectives: Check the knowledge of the users the diabetes program about preventive care for diabetic foot, identify the orientations that the patient receives about the prevention, and to observe adherence to preventive self-care procedures. Materials and methods: Exploratory research field, involving 40 diabetic type 2 and five nurses. It was conducted a physical examination of the feet of diabetics and a structured interview with nurses. The data were organized and analyzed by descriptive statistics. Results and discussion: On physical examination there were degree of mobility impaired in $52.5 \%$ of participants; inappropriate use of footwear by 85\%; removal of cuticles in $62.5 \%$. There were no significant differences in perfusion between the right and left feet, however, there was the presence of cracks and mycoses. Points of high pressure showed lower sensitivities. The orientations provided by nurses are variables, all claim to advise on the use of footwear and nail cutting, however, there is no adherence to these guidelines and miss important items as the daily examination of feet. Conclusion: Items with lower adherence are the most simple and can be corrected. There is a need proper assessment and coaching, taking into account the degree of knowledge and the ability to process information. The multiprofessional action could potentiate orientations and increase the adherence.

Keywords: Diabetes mellitus. Diabetic foot. Prevention and control. Prevention.

\section{Introdução}

Independentemente do desenvolvimento econômico, político e social de um país, o Diabetes mellitus (DM) é um importante e crescente problema de Saúde Pública. Sua prevalência, em particular a do tipo 2, está aumentando de forma exponencial e é mais encontrada nas faixas etárias avançadas, em face do aumento da expectativa de vida e do crescimento populacional. No entanto, verifica-se ampliação do número de casos na faixa etária de 20 a 45 anos. Outro fato relevante é a associação da doença com a hospitalização de seu portador, causada, na maioria das vezes, pelas complicações da DM (1).

O DM é uma doença metabólica resultante de defeitos da secreção de insulina, hormônio produzido pelo pâncreas e que é responsável pelo controle do nível de glicose no sangue. Os efeitos principais da doença são hiperglicemia crônica relativa, com alterações no metabolismo dos carboidratos, lipídios e proteínas; e as complicações macrovasculares, microvasculares e neuropáticas (2). Dentre os tipos de DM, o tipo 2 corresponde, aproximadamente, a $90 \%$ dos casos e dentre suas complicações crônicas destacam-se as lesões ulcerativas em membros inferiores (MMII) (3).

A aproximação com a assistência a portadores de DM determinou o interesse no estudo sobre o cuidado com o pé, no sentido da prevenção da úlcera do pé diabético. Esse fenômeno decorrente da neuropatia e gera perda de sensibilidade periférica tátil, térmica e dolorosa pode determinar lesões complexas que, caso não sejam tratadas, podem levar à amputação do membro (3). Ressalta-se que cerca de 10 a $25 \%$ dos portadores de DM acima de 70 anos desenvolvem lesões em MMII e destes, 14 a 24\% evoluem para amputação (4). 0 pé diabético é considerado uma consequência de infecção, 
ulceração e ou destruição dos tecidos profundos, associados a anormalidades neurológicas e a vários graus da doença vascular periférica nos MMII (5). É considerado causa comum de invalidez, já que por causa da possível amputação do membro afetado induz a diminuição da qualidade de vida do diabético (2).

A úlcera ocorre no dorso, dedos ou bordas do pé e, geralmente, está associada ao uso de calçados inadequados (2), e é mais frequente em homens devido ao mau controle das complicações crônicas (1). As causas frequentes de úlcera diabética são: biomecânica alterada; pé com sensibilidade diminuída; insuficiência arterial; incapacidade do autocuidado; e deficiência quanto às orientações aos cuidados preventivos (4).

Outro fator a ser destacado é a diminuição de sudorese que resulta em uma parede fina e ressecada, facilitando rachaduras, perda da sensibilidade e atrofia muscular. Dessa forma, surgem calosidades, microfraturas e, consequentemente, as úlceras (2).

As úlceras diabéticas podem ser neuropáticas, vasculares e mistas. As neuropáticas englobam o mal perfurante plantar resultante dos pontos de pressão, associado à diminuição da sensibilidade protetora, a qual é causada por uma calosidade plantar que acaba sendo traumática (2). As úlceras neuropáticas ocorrem em áreas de distribuição do peso e do atrito, especialmente sob as epífises distais do metatarso. As úlceras isquêmicas englobam lesões secundárias, pequenos traumas e escoriações (4).

As causas das infecções das ulcerações no pé podem ser caracterizadas como monomicrobiana ou polimicrobiana; esta última está presente em 60 a 80\% dos pacientes acometidos por infecções (6).

Sabe-se que para a prevenção adequada, tanto das úlceras plantares quanto das amputações, é necessário identificar os riscos $(1,3)$. Os locais de maior risco para lesões são os dedos, devido às deformidades; os sucos interdigitais, pelas fissuras e infecções secundárias; a região distal do pé, por infecções em proeminências dos metatarsos; e a região medial do pé, pelas calosidades e por ser uma região de apoio (7).

A prevenção é a primeira linha de defesa contra as úlceras diabéticas. Estudos têm demonstrado que programas educacionais abrangentes, que incluem exame regular dos pés, classificação de risco e educação terapêutica, podem reduzir a ocorrência de lesões nos pés em até $50 \%(8,9)$. Os diabéticos devem observar diariamente seus pés buscando a presença de edema, eritema, calosidade, descoloração, cortes ou perfurações, e secura excessiva; na impossibilidade de o portador realizar essa observação, um familiar deve procedê-la $(7,8,10)$.

Dentre os principais cuidados a serem tomados estão $(1,7)$ : restrição absoluta do fumo; exame diário dos pés, inclusive entre os dedos; lavagem dos pés com água morna, tendendo para fria; secagem cuidadosa dos pés, principalmente entre os dedos, de preferência com tecido de algodão macio; uso proibido de álcool, ou outras substâncias que ressequem a pele; uso de creme hidratante na perna e nos pés, porém, nunca entre os dedos; proibição da retirada de cutícula; corte de unhas em linha reta, sem deixar pontas e, se necessário, lixar as unhas; uso de meias de algodão sem costura, sem elásticos e preferencialmente claras; não andar descalço; uso proibido de calçados apertados, de bico fino, sandálias abertas de borracha ou plástico e contida entre os dedos; verificação da parte interna do calçado, antes de vesti-lo, a procura de objeto ou saliência que possa machucar; elevação dos pés e movimento dos dedos para melhora da circulação sanguínea; evitar o uso de bolsa de água quente; evitar exposição ao frio excessivo; e cuidados com animais domésticos e insetos.

A equipe de saúde, quando ciente do alto risco de complicações é mais propensa ao incentivo para o autocuidado dos pés de seus pacientes, mas esses profissionais devem receber educação continuada sobre o assunto. Por outro lado, os portadores necessitam ser conscientizados da importância da adesão às orientações prestadas e compreender que este é um compromisso para o resto de sua vida, pois, contribui para a sua própria qualidade de vida $(9,10)$.

No Brasil, no âmbito das políticas públicas de atenção básica à saúde, o Programa Nacional de Diabetes se caracteriza por um conjunto de ações de saúde, no âmbito individual e coletivo, que abrangem a promoção e a proteção da saúde; a prevenção de agravos; o diagnóstico, o tratamento, a reabilitação e a manutenção da saúde (11). Nesse domínio de atenção cabe à equipe multiprofissional o atendimento ao diabético, entretanto o desenvolvimento de atividades educativas e o estabelecimento de estratégias para favorecer adesão ao tratamento são de competência do profissional enfermeiro (11). No contexto do atendimento multiprofissional ressalta-se a abordagem fisioterapêutica quando da ocorrência de complicações vasculares crônicas que diminuem a capacidade funcional pelas retrações musculares e limitações articulares, bem como edemas em membros inferiores pela restrição de mobilidade que acarretam ao paciente. 
Verifica-se que o enfermeiro tem importante função na orientação dos cuidados necessários aos portadores de DM para evitar o aparecimento de lesões ulcerativas; porém, por não ser o único profissional que atende esse grupo de indivíduos e pela necessidade de orientações educativas no sentido de prevenção de úlceras, surgiu o interesse em descobrir quais as orientações oferecidas por esse profissional aos diabéticos, bem como se o portador está consciente do seu papel no autocuidado.

Nesse contexto, as questões norteadoras deste estudo foram: Qual é a atuação do enfermeiro na prevenção do pé diabético? Os portadores de DM conhecem os cuidados preventivos do pé diabético? Os portadores de DM aderem às orientações sobre os cuidados preventivos do pé diabético?

Este estudo teve como objetivos verificar o conhecimento dos usuários do programa de diabetes acerca de cuidados preventivos ao pé diabético, identificar as orientações que o paciente recebe quanto a essa prevenção, e observar nos participantes do programa de diabetes a aderência aos procedimentos de autocuidado preventivos ao pé diabético.

\section{Materiais e métodos}

Trata-se de uma pesquisa exploratória de campo, de abordagem quantitativa. Teve como sujeitos de pesquisa 40 diabéticos do tipo 2 participantes do programa e cinco enfermeiros que atuavam numa Unidade de Saúde (US) inserida no Programa de Saúde da Família de um Distrito Sanitário de Curitiba (PR). Os critérios de inclusão dos diabéticos foram: ter idade acima de 40 anos e serem participantes do programa da US por tempo mínimo de um ano. Foram excluídos os portadores de úlcera de pé diabético, os que possuíam história anterior de úlcera de pé diabético e os que tinham parte ou totalidade de um dos MMII amputados.

A coleta dos dados foi realizada em duas fases, no período entre os meses de fevereiro a abril de 2010. Foi aplicado um questionário estruturado aos enfermeiros e realizado um exame físico do pé dos diabéticos, utilizando um instrumento validado (2), que foi adaptado para este estudo. 0 exame físico foi efetivado durante os atendimentos individual ou coletivo do portador de DM, pelas acadêmicas integrantes da pesquisa, com supervisão da orientadora. Antes da coleta de dados, as alunas e a orientadora passaram por um treinamento com uma enfermeira estomaterapeuta que balizou a forma de exame físico dos pés dos diabéticos, reduzindo, assim, erros decorrentes de avaliações sustentadas por parâmetros individuais.

A avaliação dos pés foi operacionalizada com o levantamento das seguintes variáveis:

a) sinal da prece com as mãos - solicitou-se ao participante que demonstrasse qual a posição que assume quando realiza uma oração, com o intuito de identificar o grau de deformidade dos pés. Grau 1 representa a limitação de uma articulação interfalangiana; grau 2, a limitação de duas articulações interfalangianas; e grau 3, a limitação de duas articulações interfalangianas e das metacarpofalangianas;

b) higiene - momento em que se checaram sujidades aparentes. Os critérios para diferenciar uma higiene boa de regular ou irregular foram: a boa higiene refere-se ao pé que não apresenta nenhuma sujidade; a regular caracterizou-se pela presença mínima de sujidade; e a irregular era constatada pela presença visível de sujidade, desde que não fosse relacionada a um curto espaço de tempo (ex. poeira pelo andar de casa até o local da avaliação);

c) tipo dos calçados - aberto ou fechados. Se fechado, examinou-se o tipo do bico e se aberto, a presença de tiras;

d) característica das meias - identificaram-se tecido e cor;

e) hidratação - verificaram-se áreas de ressecamento, rachaduras (fissuras) e prega cutânea;

f) condição aparente dos pés - observou-se o tipo de corte nas unhas e se foram lixadas adequadamente, se entre os dedos havia umidade e se havia sinais e sintomas indicativos de micose;

g) sensibilidades - as sensibilidades protetora e tátil foram avaliadas usando Estesiômetro (Semmes-weinstein monofilamento) mediante a técnica da resposta sim-não ao toque do monofilamento de $0,05 \mathrm{~g}$ em 10 regiões do pé: primeiro (ponto 1), terceiro (ponto 2 ) e quinto (ponto 3) dígitos plantares; primeira (ponto 4), terceira (ponto 5) e quinta (ponto 6) cabeça dos metatarsos plantares; laterais esquerda (ponto 7) e direita (ponto 8) do meio plantar; calcâneo (ponto 9) e dorso (ponto 10) entre primeiro e segundo dedos (12). A sensibilidade 
térmica foi detectada com o uso de ampolas quentes e frias com soro fisiológico, nos mesmos pontos. A sensibilidade tátil e térmica foi avaliada segundo o Sistema de Classificação de Risco (10), classificado como: sem risco, baixo risco e alto risco. Essa classificação é feita pela incapacidade de sentir os estímulos feitos pelos monofilamentos ou de perceber a diferença da temperatura das ampolas de soro fisiológico em quatro ou mais pontos entre os dez pontos avaliados, caracterizando o grau de neuropatia e incapacidade protetora dos pés. Cabe ressaltar que os participantes deste estudo não foram classificados no grau 3, pois processos ulcerativos e amputações eram considerados critérios de exclusão.

Os dados obtidos foram digitados em planilha Excel $^{\circledR}$, analisados por estatística descritiva simples e discutidos à luz da literatura sobre o tema.

A pesquisa foi aprovada pelo Comitê de Ética em Pesquisa da Pontifícia Universidade Católica do Paraná (CEP/PUCPR), com parecer n. 2.929/09. Para resguardar a privacidade, todos os participantes foram examinados e/ou entrevistados individualmente, em sala separada.

\section{Resultados e discussão}

Os resultados são apresentados em duas partes: na primeira, mostram-se os dados relativos ao exame físico dos pés, correlacionando com o Consenso do Conselho Internacional do Pé Diabético (10); na segunda, comenta-se a avaliação das respostas dos Enfermeiros.

\section{Exame físico dos pés}

Dos 40 participantes, $57,5 \%$ eram do sexo feminino e $42,5 \%$ do masculino, com faixa etária entre 40 a 80 anos de idade.

Quando solicitado que posicionassem as mãos em sinal de prece, apurou-se que $47,5 \%$ dos avaliados eram caracterizados por grau $0,42,5 \%$, grau 1 , e $10 \%$, grau 2 , evidenciando que o grau de mobilidade encontra-se afetado em mais da metade dos avaliados, o que pode levar a uma limitação musculoesquelética. Os dados encontrados são semelhantes a estudo que avaliou a mobilidade em um grupo de idosas diabéticas, que aponta que a não evolução do grau pode estar relacionada aos alongamentos realizados por elas nas sessões de fisioterapia (13); isso reforça a necessidade de ação multidisciplinar para o atendimento ao diabético.

Em relação à higiene, em 70\% dos casos foi classificada como boa, e em 30\%, como regular. Neste estudo as condições de higiene foram avaliadas por exame; na literatura, encontrou-se estudo semelhante que questionou a higiene diária, sendo que $90 \%$ dos participantes afirmaram realizar higiene diária dos pés (14).

Quanto ao uso dos calçados, na população avaliada observou-se que somente 15\% faziam uso de calçados adequados, fato considerado negativo, pois em estudo semelhante foi verificado $55 \%$ de adequação (14). Ao avaliar o bico do calçado, $5 \%$ eram de bicos finos, $15 \%$ de bico quadrado e $80 \%$ de bico redondo. Sabe-se que o calçado adequado caracteriza-se pelo conforto, sem costuras e do número ideal para que não fique muito apertado, nem frouxo. Não são recomendáveis calçado de bico fino e chinelo de dedos porque causam pontos de pressão nos pés (10).

Ressalta-se que $85 \%$ dos casos de úlceras que necessitam de internação são originárias de lesões superficiais em pessoas com neuropatia periférica, lesões estas diretamente relacionadas a uso de calçados impróprios (10).

Em relação ao uso de meias, $62,5 \%$ não as utilizam. Dentre os que fazem uso da peça, $5 \%$ eram sem costuras, $30 \%$ com costura e $2,5 \%$ sintéticas. Avaliando a cor, $26,4 \%$ eram brancas e $73,3 \%$ de outras cores. Para os diabéticos há indicação de meias de algodão, sem costuras e preferencialmente claras ou na cor branca, pois facilita a visualização de possíveis lesões (10).

Quanto à hidratação dos pés, 55\% dos avaliados apresentam pés hidratados e $45 \%$ ressecados. Esse dado confirma outros estudos, um que mostrou que $45 \%$ dos pacientes usavam hidratante (14), outro que evidenciou que $54,4 \%$ das pessoas apresentavam pés hidratados (15).

Observou-se também que $62,5 \%$ dos avaliados têm o hábito de retirar cutículas; esse fato pode estar relacionado à prevalência de mulheres neste estudo. Outro dado relevante é o hábito de lixar as unhas, verificado em de $67,5 \%$ dos participantes, acima do encontrado em outro estudo que obteve $43 \%$ (14). No referente ao corte das unhas, notou-se leve diferença entre os cortes quadrado e redondo, 52,5\% e 47,5\%, respectivamente. Esse resultado difere de outro estudo (15), em que 70,7\% dos avaliados possuíam 
corte arredondado e 29,3\%, corte quadrado/reto; o resultado também se contrapõe a outra investigação (14), em que $43 \%$ dos participantes tinham o corte em linha reta. Cabe ressaltar que o corte quadrado é o indicado devido a menor possibilidade de lesão nos cantos dos dedos (10).

Não houve diferença significativa na perfusão entre os pés (esquerdo e direito), sendo $70 \%$ e $72,5 \%$ com retorno normal, respectivamente. Em relação ao tipo de pé, $50 \%$ dos avaliados possuem pé normal, $47,5 \%$ se classificam no tipo cavo, e 2,5\% como plano. Quanto aos dedos, 92,5\% apresentam tipo normal e $7,5 \%$, tipo garra, não se verificando diferença entre o pé esquerdo e direito.

A Tabela 1 mostra os resultados da avaliação da presença de umidade, micose e rachadura.

Observa-se que as condições dos pés esquerdo e direito são semelhantes e, de maneira geral, podem ser consideradas satisfatórias em relação à presença de umidade, e preocupantes em relação a rachaduras e micose. Um dos fatores desencadeantes de pé diabético são as micoses (16), e nos resultados são verificados sinais e sintomas de micoses em $27,5 \%$ no pé direito e $17,5 \%$ no esquerdo, percentual inferior ao encontrado em outro estudo que foi de $35 \%$ (15), porém não menos inquietante, necessitando de orientações quanto à hidratação e à adequada higiene.

Os resultados das avaliações das sensibilidades tátil e térmica dos pés estão apresentados nas Tabelas 2 e 3.
Os pontos de maior prevalência quanto à ausência de sensibilidade ao estímulo, tanto tátil como térmico foram os 5, 6 e 9. Os pontos citados são locais em que há altas pressões em proeminências ósseas, o ponto 5 exerce pressão contra o osso cuneiforme lateral, o ponto 6 contra o osso cuboide e o ponto 9 contra o osso calcâneo. Essas pressões estão diretamente associadas a calosidades, que são preditores da redução de sensibilidade e dos processos ulcerativos.

Esse resultado direciona o número de avaliações ou consultas necessárias ao ano para cada individuo, sendo: ausente, uma vez ao ano; grau 1, uma vez a cada 6 meses; grau 2, uma vez a cada 3 meses; e grau 3 , uma vez de 1 a 3 meses (10).

\section{Orientações realizadas pelos enfermeiros}

Embora seja de competência do enfermeiro, junto à equipe de saúde, orientar, sensibilizar e motivar as pessoas quanto às mudanças de atitude, estas, por sua vez, devem incorporar as informações recebidas (16). Os resultados deste estudo são importantes para outros profissionais de saúde, entre eles o fisioterapeuta, principalmente no que se refere ao estabelecimento da conduta de reabilitação cinético-funcional.

A conduta de reabilitação cinético-funcional visa aumentar a mobilidade articular, reduzir edemas em membros inferiores e equilibrar a musculatura envolvida na marcha, diminuindo as áreas de excesso

Tabela 1 - Distribuição da presença de umidade, micoses e rachadura, segundo avaliação dos pés direito e esquerdo dos diabéticos $(n=40)$

\begin{tabular}{llcc}
\hline & Variável & Pé direito (\%) & Pé esquerdo (\%) \\
\hline \multirow{2}{*}{ Umidade } & Bromidose & 5,0 & 7,5 \\
& Normal & 95,0 & 92,5 \\
\hline \multirow{2}{*}{ Micose } & Sim & 27,5 & 17,5 \\
& Não & 72,5 & 82,5 \\
\hline \multirow{4}{*}{ Rachaduras } & Calcanhar & 10,0 & 17,5 \\
& Calcanhar /lateral & 7,5 & 7,5 \\
& Lateral & 5,0 & 2,5 \\
& Plantar & 5,0 & 5,0 \\
& Não tem & 72,5 & 67,5 \\
\hline
\end{tabular}

Fonte: Dados da pesquisa. 
Tabela 2 - Distribuição de pontos não sensíveis, com respectiva frequência de pessoas, relacionada às sensibilidades tátil e térmica, dos pés esquerdo e direito $(n=40)$

\begin{tabular}{cccccccc}
\hline & \multicolumn{2}{c}{ Sensibilidade tátil } & \multicolumn{3}{c}{ Sensibilidade térmica } \\
\multicolumn{2}{c}{ Pé esquerdo } & \multicolumn{2}{c}{ Pé direito } & \multicolumn{2}{c}{ Pé esquerdo } & \multicolumn{2}{c}{ Pé direito } \\
\hline $\begin{array}{c}\text { Pontos não } \\
\text { sensíveis }\end{array}$ & $\begin{array}{c}\text { Número de } \\
\text { pessoas }\end{array}$ & $\begin{array}{c}\text { Pontos não } \\
\text { sensíveis }\end{array}$ & $\begin{array}{c}\text { Número de } \\
\text { pessoas }\end{array}$ & $\begin{array}{c}\text { Pontos não } \\
\text { sensíveis }\end{array}$ & $\begin{array}{c}\text { Número de } \\
\text { pessoas }\end{array}$ & $\begin{array}{c}\text { Pontos não } \\
\text { sensiveis }\end{array}$ & $\begin{array}{c}\text { Número de } \\
\text { pessoas }\end{array}$ \\
$0 \%$ & 31 & $0 \%$ & 24 & $0 \%$ & 29 & $0 \%$ & 27 \\
$40 \%$ & 4 & $40 \%$ & 8 & $40 \%$ & 3 & $40 \%$ & 4 \\
$50 \%$ & 1 & $50 \%$ & 3 & $50 \%$ & 1 & $50 \%$ & 2 \\
$60 \%$ & 1 & $60 \%$ & 2 & $60 \%$ & 3 & $60 \%$ & 2 \\
$80 \%$ & 4 & $80 \%$ & 2 & $70 \%$ & 2 & $70 \%$ & 2 \\
& & $90 \%$ & 1 & $80 \%$ & 1 & $100 \%$ & 4 \\
& & $100 \%$ & 1 & $100 \%$ & 2 & & \\
\hline
\end{tabular}

Fonte: Dados da pesquisa.

Tabela 3 - Distribuição da frequência absoluta da classificação do grau de risco em relação à avaliação de sensibilidade tátil e térmica dos pés esquerdo e direito $(n=40)$

\begin{tabular}{lcccc}
\hline & Tipo de Sensibilidade & & Classificação do Grau de Risco & \\
& & Sem Risco & Baixo Risco & Alto Risco \\
\hline \multirow{2}{*}{ Tátil } & PE & 8 & 22 & 10 \\
& PD & 10 & 13 & 17 \\
\hline \multirow{2}{*}{ Térmica } & PE & 8 & 20 & 12 \\
& PD & 10 & 16 & 0 \\
\hline
\end{tabular}

Legenda: $\mathrm{PE}=$ pé esquerdo; $\mathrm{PD}=$ pé direito.

Fonte: Dados da pesquisa.

de pressão plantar $(17,18)$. A adesão por parte do diabético às orientações oferecidas pelo Enfermeiro ajudará o Fisioterapeuta a promover um maior nível de independência funcional e retorno às atividades de vida diária com segurança, com consequente reflexo no comparecimento às atividades promovidas pela US.

Os cinco Enfermeiros entrevistados afirmam orientar os usuários no que se refere à restrição de fumo, ao uso de calçados, ao corte de unhas e recomendar que procurem pelo serviço quando houver qualquer alteração na pele.

É importante orientar os usuários também sobre lavagem diária dos pés com água morna, uso de bolsas de água, exposição ao frio excessivo, presença de animais domésticos, elevação dos pés, e uso de álcool nos pés. A respeito dessas questões, os enfermeiros afirmam orientar raramente. No que se refere aos itens andar descalço, uso de sandálias, retirada de cutículas, pés imersos em água quente, exposição ao frio, os Enfermeiros afirmam que não fazem orientação.

Os Enfermeiros indicam que às vezes informam os pacientes sobre questões de extrema importância como exame diário dos pés, uso de sandálias de borracha, presença de animais domésticos para evitar ferimentos e não usar álcool nos pés. Esses itens deveriam ser reforçados em todas as consultas.

Ao comparar as respostas dos Enfermeiros com a avaliação dos pés dos 40 participantes, notou-se 
que, quando a orientação sobre o uso de calçados adequados é abordada, o Enfermeiro afirma que realiza esta orientação em $100 \%$ de seus encontros com os diabéticos. Entretanto, $85 \%$ dos participantes fazem uso de calçados inadequados, com bico fino, salto, sapatos secos, apertados ou muito frouxos, com destaque para o uso em grande escala de chinelos de dedo de borracha ou similar. Esse resultado leva à discussão da presença de duas hipóteses: a orientação não está sendo realmente realizada pelo Enfermeiro ou o usuário não tem acesso a outro tipo de calçado, independentemente da orientação que tenha recebido.

Quanto ao corte das unhas, todos os enfermeiros afirmam orientar, mas, ao comparar com o exame, $52,5 \%$ realizam o corte correto e $47,5 \%$ usam o corte redondo, que é considerado inadequado.

Quatro dos cinco Enfermeiros responderam que orientam sobre a retirada das cutículas. Ao comparar esse dado com o exame, nota-se que $82,5 \%$ retiram as cutículas. Considera-se que a prevalência do sexo feminino pode interferir nesse resultado. No entanto, discute-se a eficácia da orientação, pois um grande percentual de mulheres não as segue.

\section{Conclusão}

Este estudo demonstrou que, com relação às características avaliadas, apresentam-se como inadequadas a retirada da cutícula, o uso de calçados, e o tipo e cor das meias. Ao avaliar a coleta de dados, nota-se que há um alto percentual de pessoas que realizam o corte das unhas de forma inadequada.

Ao comparar os questionários respondidos pelos Enfermeiros e o exame realizado nos participantes, constatou-se que os pacientes, por diferentes motivos, não aderem a algumas orientações. Conclui-se que os itens com menor adesão são os mais simples, baratos e passíveis de correção. São relevantes, portanto, adequada avaliação e acompanhamento individual, levando em consideração o grau de conhecimento e a facilidade para processar as informações.

Entendendo que uma ação multiprofissional, com esforço coletivo, poderia potencializar as orientações e aumentar a aderência às mesmas, os resultados do presente estudo poderão subsidiar a ação de enfermeiros e fisioterapeutas no estabelecimento de condutas apropriadas para prevenção de lesões que determinam a morbidade de úlcera de pé diabético.

\section{Referências}

1. Milech A. Pé diabético. In: Oliveira JEP, Milech A, organizadores. Diabetes Mellitus: clínica, diagnóstico, tratamento multidisciplinar. São Paulo: Atheneu; 2004. p. 7-14.

2. Parisi MCR. Úlceras no Pé Diabético. In: Jorge AS, Dantas SRPE, organizadores. Abordagem multidisciplinar do tratamento de feridas. São Paulo: Atheneu; 2005. p. 279-86.

3. Gomes MB, Cobas R. Diabetes mellitus. In: Gossi SAA, Pascali PM, organizadores. Cuidados de enfermagem em Diabetes mellitus. São Paulo: Sociedade Brasileira de Diabetes; 2009. p. 6-17.

4. Irion G. Feridas: novas abordagens, manejo clínico e atlas em cores. Rio de Janeiro: Guanabara Koogan; 2005.

5. Nunes PAM, Resende KF, Castro AA, Pitta GBB, Figueiredo LFP, Miranda F Júnior. Fatores predisponentes para amputação de membro inferior em pacientes diabéticos internados com pés ulcerados no estado de Sergipe. J Vasc Bras. 2006;5(2):123-30.

6. Carvalho CBM, Neto RM, Aragão LP, Oliveira MM, Nogueira MB, Forti AC. Pé diabético: análise bacteriológica de 141 casos. Arq Bras Endocrinol Metab. 2004;48(3): 406-13.

7. Pace AE, Carvalho VF. Cuidados de enfermagem na prevenção de complicações nos pés nas pessoas com diabetes mellitus. In: Gossi SAA, Pascali PM. Cuidados de enfermagem em Diabetes mellitus. São Paulo: Sociedade Brasileira de Diabetes; 2009. p. 124-37.

8. Gogia PP. Feridas: tratamento e cicatrização. Rio de Janeiro: Revinter; 2003.

9. Cosson ICO, Ney-Oliveira F, Adan LF. Avaliação do conhecimento de medidas preventivas do pé diabético em pacientes de Rio Branco, Acre. Arq Bras Endocrinol Metab. 2005; 49(4):548-56.

10. Grupo de trabalho internacional sobre pé diabético. Consenso internacional sobre pé diabético. Brasília: Secretaria de Estado de Saúde do Distrito Federal; 2001.

11. Ministério da Saúde (Brasil). Cadernos de atenção básica Diabetes Mellitus. Brasília: Ministério da Saúde; 2006. 
12. Ochoa-Vigo K, Pace AE. Pé diabético: estratégias para prevenção. Acta Paul Enferm. 2005;18(1):100-9.

13. Gomes EB, Souza KNS, Paz SN. Avaliação da força de preensão palmar em idosas diabéticas e não diabéticas e sua correlação com o sinal da prece. Brasília Med. 2009;46(4):317-23.

14. Andrade NHS, Dal Sasso-Mendes K, Faria HTG, Martins TA, Santos MA, Teixeira CRS, et al. Pacientes com diabetes mellitus: cuidados e prevenção do pé diabético em atenção primária à saúde. Rev enferm UERJ. 2010;18(4):616-21.

15. Amaral SA, Tavares SMD. Cuidados com os pés: conhecimento entre pessoas com diabetes mellitus. Rev eletr enf. 2009 [acesso 20 ago 2013];11(4):801-10. Disponível em: http://www.fen.ufg.br/fen_revista/ v11/n4/pdf/v11n4a05.pdf

16. Pace AE, Ochoa-Vigo K, Foss MC, Hayashida M. Fatores de risco para complicações em extremidades inferiores de pessoas com Diabetes Mellitus. Rev bras enferm. 2002;55(5):514-21.

17. Barros MFA; Mendes JC; Nascimento JA; Carvalho AGC. Impacto de intervenção fisioterapêutica na prevenção do pé diabético. Fisioter mov. 2012;25(4): 747-57.

18. Mendonca SS; Morais JS; Moura MCGG. Proposta de um protocolo de avaliação fisioterapêutica para os pés de diabéticos. Fisioter mov. 2011;24(2):285-98.

Recebido: 26/10/2012

Received: 10/26/2012

Aprovado: $17 / 04 / 2013$

Approved: 04/17/2013 